\title{
Observation of the semimuonic decay $D^{+} \rightarrow \omega \mu^{+} \nu_{\mu}$
}

M. Ablikim, ${ }^{1}$ M. N. Achasov, ${ }^{10, e}$ P. Adlarson,${ }^{64}$ S. Ahmed,${ }^{15}$ M. Albrecht,${ }^{4}$ A. Amoroso, ${ }^{63 a, 63 c}$ Q. An, ${ }^{60,48}$ Anita, ${ }^{21}$ Y. Bai,${ }^{47}$ O. Bakina, ${ }^{29}$ R. Baldini Ferroli, ${ }^{23 a}$ I. Balossino, ${ }^{24 a}$ Y. Ban,${ }^{38, m}$ K. Begzsuren, ${ }^{26}$ J. V. Bennett, ${ }^{5}$ N. Berger, ${ }^{28}$ M. Bertani, ${ }^{23 a}$ D. Bettoni, ${ }^{24 a}$ F. Bianchi, ${ }^{63 a, 63 c}$ J. Biernat, ${ }^{64}$ J. Bloms,${ }^{57}$ A. Bortone,${ }^{63 a, 63 c}$ I. Boyko ${ }^{29}$ R. A. Briere,${ }^{5}$ H. Cai ${ }^{65}$ X. Cai,${ }^{1,48}$ A. Calcaterra, ${ }^{23 a}$ G. F. Cao, ${ }^{1,52}$ N. Cao, ${ }^{1,52}$ S. A. Cetin, ${ }^{51 b}$ J. F. Chang, ${ }^{1,48}$ W. L. Chang, ${ }^{1,52}$ G. Chelkov, ${ }^{29, c, d}$ D. Y. Chen, ${ }^{6}$ G. Chen, ${ }^{1}$ H. S. Chen, ${ }^{1,52}$ M. L. Chen, ${ }^{1,48}$ S. J. Chen,${ }^{36}$ X. R. Chen, ${ }^{25}$ Y. B. Chen, ${ }^{1,48}$ W. Cheng, ${ }^{63 \mathrm{c}}$ G. Cibinetto, ${ }^{24 \mathrm{a}}$

F. Cossio, ${ }^{63 c}$ X. F. Cui, ${ }^{37}$ H. L. Dai, ${ }^{1,48}$ J. P. Dai ${ }^{42, i}$ X. C. Dai,${ }^{1,52}$ A. Dbeyssi ${ }^{15}$ R. B. de Boer, ${ }^{4}$ D. Dedovich,${ }^{29}$ Z. Y. Deng, ${ }^{1}$ A. Denig, ${ }^{28}$ I. Denysenko, ${ }^{29}$ M. Destefanis,${ }^{63 a, 63 c}$ F. De Mori ${ }^{63 a, 63 c}$ Y. Ding, ${ }^{34}$ C. Dong, ${ }^{37}$ J. Dong, ${ }^{1,48}$ L. Y. Dong, ${ }^{1,52}$ M. Y. Dong, ${ }^{1,48,52}$ S. X. Du, ${ }^{68}$ J. Fang, ${ }^{1,48}$ S. S. Fang, ${ }^{1,52}$ Y. Fang, ${ }^{1}$ R. Farinelli, ${ }^{24 a, 24 b}{ }^{2}$ L. Fava, ${ }^{63 b, 63 c}$ F. Feldbauer, ${ }^{4}$ G. Felici, ${ }^{23 a}$ C. Q. Feng, ${ }^{60,48}$ M. Fritsch, ${ }^{4}$ C. D. Fu, ${ }^{1}$ Y. Fu, ${ }^{1}$ X. L. Gao, ${ }^{60,48}$ Y. Gao,${ }^{61}$ Y. Gao, ${ }^{38, m}$ Y. G. Gao, ${ }^{6}$ I. Garzia, ${ }^{24 a, 24 b}$ E. M. Gersabeck, ${ }^{55}$ A. Gilman, ${ }^{56}$ K. Goetzen, ${ }^{11}$ L. Gong, ${ }^{37}$ W. X. Gong, ${ }^{1,48}$ W. Gradl, ${ }^{28}$ M. Greco, ${ }^{63 a, 63 c}$ L. M. Gu, ${ }^{36}$ M. H. Gu, ${ }^{1,48}$ S. Gu, ${ }^{2}$ Y. T. Gu, ${ }^{13}$ C. Y. Guan, ${ }^{1,52}$ A. Q. Guo, ${ }^{22}$ L. B. Guo, ${ }^{35}$ R. P. Guo ${ }^{40}$ Y. P. Guo, ${ }^{28}$ Y. P. Guo, ${ }^{9, j}$ A. Guskov ${ }^{29}$ S. Han,${ }^{65}$ T. T. Han ${ }^{41}$ T. Z. Han, ${ }^{9, j}$ X. Q. Hao, ${ }^{16}$ F. A. Harris,${ }^{53}$ K. L. He,${ }^{1,52}$ F. H. Heinsius,${ }^{4}$ T. Held, ${ }^{4}$ Y. K. Heng, ${ }^{1,48,52}$ M. Himmelreich, ${ }^{11, h}$ T. Holtmann, ${ }^{4}$ Y. R. Hou, ${ }^{52}$ Z. L. Hou,${ }^{1}$ H. M. Hu, ${ }^{1,52}$ J. F. Hu, ${ }^{42, \mathrm{i}}$ T. Hu, ${ }^{1,48,52}$ Y. Hu, ${ }^{1}$ G. S. Huang ${ }^{60,48}$ L. Q. Huang, ${ }^{61}$ X. T. Huang, ${ }^{41}$ N. Huesken, ${ }^{57}$ T. Hussain, ${ }^{62}$ W. Ikegami Andersson, ${ }^{64}$ W. Imoehl, ${ }^{22}$ M. Irshad, ${ }^{60,48}$ S. Jaeger, ${ }^{4}$ S. Janchiv,${ }^{26,1}$ Q. Ji, ${ }^{1}$ Q. P. Ji, ${ }^{16}$ X. B. Ji, ${ }^{1,52}$ X. L. Ji, ${ }^{1,48}$ H. B. Jiang, ${ }^{41}$ X. S. Jiang, ${ }^{1,48,52}$ X. Y. Jiang, ${ }^{37}$ J. B. Jiao, ${ }^{41}$ Z. Jiao, ${ }^{18}$ S. Jin,${ }^{36}$ Y. Jin,${ }^{54}$ T. Johansson, ${ }^{64}$ N. Kalantar-Nayestanaki,${ }^{31}$ X. S. Kang, ${ }^{34}$ R. Kappert ${ }^{31}$ M. Kavatsyuk, ${ }^{31}$ B. C. Ke, ${ }^{43,1}$ I. K. Keshk, ${ }^{4}$ A. Khoukaz, ${ }^{57}$ P. Kiese,${ }^{28}$ R. Kiuchi, ${ }^{1}$ R. Kliemt, ${ }^{11}$ L. Koch, ${ }^{30}$ O. B. Kolcu, ${ }^{51 b, g}$ B. Kopf, ${ }^{4}$ M. Kuemmel, ${ }^{4}$ M. Kuessner, ${ }^{4}$ A. Kupsc, ${ }^{64}$ M. G. Kurth, ${ }^{1,52}$ W. Kühn, ${ }^{30}$ J. J. Lane, ${ }^{55}$ J. S. Lange, ${ }^{30}$ P. Larin,${ }^{15}$ L. Lavezzi, ${ }^{63 \mathrm{c}}$ H. Leithoff, ${ }^{28}$ M. Lellmann, ${ }^{28}$ T. Lenz, ${ }^{28}$ C. Li, ${ }^{39}$ C. H. Li ${ }^{33}$ Cheng Li ${ }^{60,48}$ D. M. Li, ${ }^{68} \mathrm{~F} . \mathrm{Li}^{1,}, 48 \mathrm{G}$. Li, ${ }^{1}$ H. B. Li, ${ }^{1,52}$ H. J. Li, ${ }^{9, j}$ J. L. Li ${ }^{41}$ J. Q. Li, ${ }^{4}$ Ke Li, ${ }^{1}$ L. K. Li, ${ }^{1}$ Lei Li, ${ }^{3}$ P. L. Li, ${ }^{60,48}$ P. R. Li, ${ }^{32}$ S. Y. Li,${ }^{50}$ W. D. Li, ${ }^{1,52}$ W. G. Li, ${ }^{1}$ X. H. Li, ${ }^{60,48}$ X. L. Li, ${ }^{41}$ Z. B. Li ${ }^{49}$ Z. Y. Li, ${ }^{49}$ H. Liang, ${ }^{1,52}$ H. Liang, ${ }^{60,48}$ Y. F. Liang, ${ }^{45}$ Y. T. Liang, ${ }^{25}$ L. Z. Liao, ${ }^{1,52}$ J. Libby, ${ }^{21}$ C. X. Lin ${ }^{49}$ B. Liu, ${ }^{42, i}$ B. J. Liu, ${ }^{1}$ C. X. Liu, ${ }^{1}$ D. Liu, ${ }^{60,48}$ D. Y. Liu, ${ }^{42, i}$ F. H. Liu, ${ }^{44}$ Fang Liu, ${ }^{1}$ Feng Liu, ${ }^{6}$ H. B. Liu, ${ }^{13}$ H. M. Liu, ${ }^{1,52}$ Huanhuan Liu, ${ }^{1}$ Huihui Liu, ${ }^{17}$ J. B. Liu, ${ }^{60,48}$ J. Y. Liu, ${ }^{1,52}$ K. Liu, ${ }^{1}$ K. Y. Liu, ${ }^{34}$ Ke Liu, ${ }^{6}$ L. Liu, ${ }^{60,48}$ L. Y. Liu, ${ }^{13}$ Q. Liu, ${ }^{52}$ S. B. Liu, ${ }^{60,48}$ Shuai Liu, ${ }^{46}$ T. Liu, ${ }^{1,52}$ X. Liu, ${ }^{32}$ Y. B. Liu ${ }^{37}$ Z. A. Liu, ${ }^{1,48,52}$ Z. Q. Liu, ${ }^{41}$ Y. F. Long, ${ }^{38, m}$ X. C. Lou, ${ }^{1,48,52}$ F. X. Lu, ${ }^{16}$ H. J. Lu, ${ }^{18}$ J. D. Lu, ${ }^{1,52}$ J. G. Lu, ${ }^{1,48}$ X. L. Lu, ${ }^{1}$ Y. Lu, ${ }^{1}$ Y. P. Lu, ${ }^{1,48}$ C. L. Luo, ${ }^{35}$ M. X. Luo, ${ }^{67}$ P. W. Luo, ${ }^{49}$ T. Luo, ${ }^{9, j}$ X. L. Luo, ${ }^{1,48}$ S. Lusso, ${ }^{63 c}$ X. R. Lyu ${ }^{52}$ F. C. Ma ${ }^{34}$ H. L. Ma ${ }^{1}$ L. L. Ma, ${ }^{41}$ M. M. Ma, ${ }^{1,52}$ Q. M. Ma, ${ }^{1}$ R. Q. Ma ${ }^{1,52}$ R. T. Ma ${ }^{52}$ X. N. Ma ${ }^{37}$ X. X. Ma,${ }^{1,52}$ X. Y. Ma, ${ }^{1,48}$ Y. M. Ma, ${ }^{41}$ F. E. Maas, ${ }^{15}$ M. Maggiora, ${ }^{63 a, 63 c}$ S. Maldaner, ${ }^{28}$ S. Malde, ${ }^{58}$ Q. A. Malik, ${ }^{62}$ A. Mangoni, ${ }^{23 b}$ Y. J. Mao ${ }^{38, m}$ Z. P. Mao, ${ }^{1}$ S. Marcello, ${ }^{63 a, 63 c}$ Z. X. Meng, ${ }^{54}$ J. G. Messchendorp, ${ }^{31}$ G. Mezzadri, ${ }^{24 a}$ T. J. Min, ${ }^{36}$ R. E. Mitchell, ${ }^{22}$ X. H. Mo, ${ }^{1,48,52}$ Y. J. Mo, ${ }^{6}$ N. Yu. Muchnoi, ${ }^{10, e}$ H. Muramatsu, ${ }^{56}$ S. Nakhoul, ${ }_{11, h}$ Y. Nefedov, ${ }^{29}$ F. Nerling, ${ }^{11, h}$ I. B. Nikolaev, ${ }^{10, \mathrm{e}}$ Z. Ning, ${ }^{1,48}$ S. Nisar, ${ }^{8, \mathrm{k}}$ S. L. Olsen, ${ }^{52}$ Q. Ouyang, ${ }^{1,48,52}$ S. Pacetti, ${ }^{23 b}$ X. Pan, ${ }^{46}$ Y. Pan, ${ }^{55}$ M. Papenbrock,${ }^{64}$ A. Pathak, ${ }^{1}$ P. Patteri, ${ }^{23 a}$ M. Pelizaeus, ${ }^{4}$ H. P. Peng,${ }^{60,48}$ K. Peters, ${ }^{11, h}$ J. Pettersson, ${ }^{64}$ J. L. Ping, ${ }^{35}$ R. G. Ping, ${ }^{1,52}$ A. Pitka, ${ }^{4}$ R. Poling, ${ }^{56}$ V. Prasad, ${ }^{60,48}$ H. Qi,${ }^{60,48}$ H. R. Qi ${ }^{50}$ M. Qi ${ }^{36}$ T. Y. Qi, ${ }^{2}$ S. Qian, ${ }^{1,48}$ C. F. Qiao, ${ }^{52}$ L. Q. Qin, ${ }^{12}$ X. P. Qin, ${ }^{13}$ X. S. Qin, ${ }^{4}$ Z. H. Qin, ${ }^{1,48}$ J. F. Qiu, ${ }^{1}$ S. Q. Qu ${ }^{37}$ K. H. Rashid, ${ }^{62}$

K. Ravindran, ${ }^{21}$ C. F. Redmer, ${ }^{28}$ A. Rivetti, ${ }^{63 \mathrm{c}}$ V. Rodin, ${ }^{31}$ M. Rolo, ${ }^{63 \mathrm{c}}$ G. Rong, ${ }^{1,52}$ Ch. Rosner, ${ }^{15} \mathrm{M}$. Rump, ${ }^{57}$ A. Sarantsev, ${ }^{29, \mathrm{f}}$ M. Savrié, ${ }^{24 \mathrm{~b}}$ Y. Schelhaas, ${ }^{28}$ C. Schnier, ${ }^{4}$ K. Schoenning, ${ }^{64}$ D. C. Shan, ${ }^{46}$ W. Shan, ${ }^{19}$ X. Y. Shan, ${ }^{60,48}$ M. Shao, ${ }^{60,48}$ C. P. Shen, ${ }^{2}$ P. X. Shen, ${ }^{37}$ X. Y. Shen, ${ }^{1,52}$ H. C. Shi,${ }^{60,48}$ R. S. Shi, ${ }^{1,52}$ X. Shi, ${ }^{1,48}$ X. D. Shi, ${ }^{60,48}$ J. J. Song, ${ }^{41}$ Q. Q. Song, ${ }^{60,48}$ W. M. Song, ${ }^{27}$ Y. X. Song,${ }^{38, \mathrm{~m}}$ S. Sosio, ${ }^{63 a, 63 c}$ S. Spataro, ${ }^{63 a, 63 c}$ F. F. Sui, ${ }^{41}$ G. X. Sun, ${ }^{1}$ J. F. Sun, ${ }^{16}$ L. Sun ${ }^{65}$

S.S. Sun, ${ }^{1,52}$ T. Sun ${ }^{1,52}$ W. Y. Sun, ${ }^{35}$ Y. J. Sun, ${ }^{60,48}$ Y. K. Sun, ${ }^{60,48}$ Y.Z. Sun, ${ }^{1}$ Z. T. Sun, ${ }^{1}$ Y. X. Tan, ${ }^{60,48}$ C. J. Tang ${ }_{36}{ }^{45}$ G. Y. Tang, ${ }^{1}$ J. Tang ${ }^{49}$ V. Thoren, ${ }^{64}$ B. Tsednee, ${ }^{26}$ I. Uman, ${ }^{51 d}$ B. Wang, ${ }^{1}$ B. L. Wang ${ }_{1,52}^{52}$ C. W. Wang, ${ }^{36}$ D. Y. Wang, ${ }_{38, m}$ H. P. Wang, ${ }^{1,52}$ K. Wang, ${ }^{1,48}$ L. L. Wang, ${ }^{1}$ M. Wang, ${ }^{41}$ M. Z. Wang, ${ }^{38, m}{ }^{15}$ Meng Wang, ${ }^{1,52}$ W. P. Wang, ${ }^{60,48}$ X. Wang, ${ }^{38, m}$

X. F. Wang, ${ }^{32}$ X. L. Wang, ${ }^{9, j}$ Y. Wang, ${ }^{60,48}$ Y. Wang, ${ }^{49}$ Y. D. Wang, ${ }^{15}$ Y. F. Wang, ${ }^{1,48,52}$ Y. Q. Wang, ${ }^{1}$ Z. Wang, ${ }^{1,48}$

Z. Y. Wang, ${ }^{1}$ Ziyi Wang, ${ }^{52}$ Zongyuan Wang, ${ }^{1,52}$ T. Weber, ${ }^{4}$ D. H. Wei, ${ }^{12}$ P. Weidenkaff, ${ }^{28}$ F. Weidner, ${ }^{57}$ H. W. Wen, ${ }^{35, a}$ S. P. Wen, ${ }^{1}$ D. J. White, ${ }^{55}$ U. Wiedner, ${ }^{4}$ G. Wilkinson, ${ }^{58}$ M. Wolke,${ }^{64}$ L. Wollenberg, ${ }^{4}$ J. F. Wu ${ }^{1,52}$ L. H. Wu, ${ }^{1}$ L. J. Wu, ${ }^{1,52}$ X. Wu, ${ }^{9, j}$ Z. Wu ${ }^{1,48}$ L. Xia ${ }^{60,48}$ H. Xiao, ${ }^{9, j}$ S. Y. Xiao, ${ }^{1}$ Y. J. Xiao, ${ }^{1,52}$ Z. J. Xiao ${ }^{35}$ Y. G. Xie, ${ }^{1,48}$ Y. H. Xie, ${ }^{6}$ T. Y. Xing, ${ }^{1,52}$ X. A. Xiong, ${ }^{1,52}$ G. F. Xu, ${ }^{1}$ J. J. Xu, ${ }^{36}$ Q. J. Xu ${ }^{14}$ W. Xu,${ }^{1,52}$ X. P. Xu, ${ }^{46}$ L. Yan ${ }^{63 a, 63 c}$ L. Yan,,${ }^{, j}$ W. B. Yan ${ }^{60,48}$ W. C. Yan ${ }^{68}$ Xu Yan, ${ }^{46}$ H. J. Yang, ${ }^{42, i}$ H. X. Yang, ${ }^{1}$ L. Yang, ${ }^{65}$ R. X. Yang ${ }^{60,48}$ S. L. Yang, ${ }^{1,52}$ Y. H. Yang, ${ }^{36}$ Y. X. Yang, ${ }^{12}$ Yifan Yang, ${ }^{1,52}$ Zhi Yang, ${ }^{25}$ M. Ye, ${ }^{1,48}$ M. H. Ye, ${ }^{7}$ J. H. Yin, ${ }^{1}$ Z. Y. You, ${ }^{49}$ B. X. Yu, ${ }^{1,48,52}$ C. X. Yu, ${ }^{37}$ G. Yu, ${ }^{1,52}$ J. S. Yu, ${ }^{20, n}$ T. Yu, ${ }^{61}$

C. Z. Yuan, ${ }^{1,52}$ W. Yuan, ${ }^{63,63 c}$ X. Q. Yuan, ${ }^{38, m}$ Y. Yuan, ${ }^{1}$ C. X. Yue, ${ }^{33}$ A. Yuncu, ${ }^{51 b, b}$ A. A. Zafar, ${ }^{62}$ Y. Zeng, ${ }^{20, n}$

B. X. Zhang, ${ }^{1}$ Guangyi Zhang, ${ }^{16}$ H. H. Zhang, ${ }^{49}$ H. Y. Zhang, ${ }^{1,48}$ J. L. Zhang, ${ }^{66}$ J. Q. Zhang, ${ }^{4}$ J. W. Zhang, ${ }^{1,48,52}$ J. Y. Zhang, ${ }^{1}$ J. Z. Zhang, ${ }^{1,52}$ Jianyu Zhang, ${ }_{58}^{1,52}$ Jiawei Zhang, ${ }^{1,52}$ L. Zhang, ${ }^{1}$ Lei Zhang, ${ }^{36}$ S. Zhang, ${ }^{49}$ S. F. Zhang, ${ }^{36}$ T. J. Zhang, ${ }^{42, i}$ X. Y. Zhang, ${ }^{41}$ Y. Zhang, ${ }^{58}$ Y. H. Zhang, ${ }^{1,48}$ Y. T. Zhang, ${ }^{60,48}$ Yan Zhang, ${ }^{60,48}$ Yao Zhang, ${ }^{1}$ Yi Zhang, ${ }^{9, j}$ 
Z. H. Zhang, ${ }^{6}$ Z. Y. Zhang, ${ }^{65}$ G. Zhao, ${ }^{1}$ J. Zhao, ${ }^{33}$ J. Y. Zhao, ${ }^{1,52}$ J. Z. Zhao, ${ }^{1,48}$ Lei Zhao, ${ }^{60,48}$ Ling Zhao, ${ }^{1}$ M. G. Zhao, ${ }^{37}$ Q. Zhao, ${ }^{1}$ S. J. Zhao, ${ }^{68}$ Y. B. Zhao, ${ }^{1,48}$ Y. X. Zhao Zhao, ${ }^{25}$ Z. G. Zhao, ${ }^{60,48}$ A. Zhemchugov, ${ }^{29, c}$ B. Zheng, ${ }^{61}$ J. P. Zheng, ${ }^{1,48}$ Y. Zheng, ${ }^{38, m}$ Y. H. Zheng, ${ }^{52}$ B. Zhong, ${ }^{35}$ C. Zhong, ${ }^{61}$ L. P. Zhou, ${ }^{1,52}$ Q. Zhou, ${ }^{1,52}$ X. Zhou, ${ }^{65}$ X. K. Zhou, ${ }^{52}$ X. R. Zhou, ${ }^{60,48}$

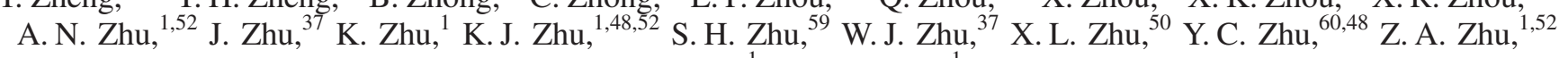
B. S. Zou, ${ }^{1}$ and J. H. Zou ${ }^{1}$

\section{(BESIII Collaboration)}

${ }^{1}$ Institute of High Energy Physics, Beijing 100049, People's Republic of China

${ }^{2}$ Beihang University, Beijing 100191, People's Republic of China

${ }^{3}$ Beijing Institute of Petrochemical Technology, Beijing 102617, People's Republic of China

${ }^{4}$ Bochum Ruhr-University, D-44780 Bochum, Germany

${ }^{5}$ Carnegie Mellon University, Pittsburgh, Pennsylvania 15213, USA

${ }^{6}$ Central China Normal University, Wuhan 430079, People's Republic of China

${ }^{7}$ China Center of Advanced Science and Technology, Beijing 100190, People's Republic of China

${ }^{8}$ COMSATS University Islamabad, Lahore Campus, Defence Road,

Off Raiwind Road, 54000 Lahore, Pakistan

${ }^{9}$ Fudan University, Shanghai 200443, People's Republic of China

${ }^{10}$ G.I. Budker Institute of Nuclear Physics SB RAS (BINP), Novosibirsk 630090, Russia

${ }^{11}$ GSI Helmholtzcentre for Heavy Ion Research GmbH, D-64291 Darmstadt, Germany

${ }^{12}$ Guangxi Normal University, Guilin 541004, People's Republic of China

${ }^{13}$ Guangxi University, Nanning 530004, People's Republic of China

${ }^{14}$ Hangzhou Normal University, Hangzhou 310036, People's Republic of China

${ }^{15}$ Helmholtz Institute Mainz, Johann-Joachim-Becher-Weg 45, D-55099 Mainz, Germany

${ }^{16}$ Henan Normal University, Xinxiang 453007, People's Republic of China

${ }^{17}$ Henan University of Science and Technology, Luoyang 471003, People's Republic of China

${ }^{18}$ Huangshan College, Huangshan 245000, People's Republic of China

${ }^{19}$ Hunan Normal University, Changsha 410081, People's Republic of China

${ }^{20}$ Hunan University, Changsha 410082, People's Republic of China

${ }^{21}$ Indian Institute of Technology Madras, Chennai 600036, India

${ }^{22}$ Indiana University, Bloomington, Indiana 47405, USA

${ }^{23 a}$ INFN Laboratori Nazionali di Frascati, I-00044 Frascati, Italy

${ }^{23 \mathrm{~b}}$ INFN and University of Perugia, I-06100 Perugia, Italy

${ }^{24 a}$ INFN Sezione di Ferrara, I-44122 Ferrara, Italy

${ }^{24 \mathrm{~b}}$ University of Ferrara, I-44122 Ferrara, Italy

${ }^{25}$ Institute of Modern Physics, Lanzhou 730000, People's Republic of China

${ }^{26}$ Institute of Physics and Technology, Peace Avenue 54B, Ulaanbaatar 13330, Mongolia

${ }^{27}$ Jilin University, Changchun 130012, People's Republic of China

${ }^{28}$ Johannes Gutenberg University of Mainz, Johann-Joachim-Becher-Weg 45, D-55099 Mainz, Germany

${ }^{29}$ Joint Institute for Nuclear Research, 141980 Dubna, Moscow region, Russia

${ }^{30}$ Justus-Liebig-Universitaet Giessen, II. Physikalisches Institut,

Heinrich-Buff-Ring 16, D-35392 Giessen, Germany

${ }^{31}$ KVI-CART, University of Groningen, NL-9747 AA Groningen, Netherlands

${ }^{32}$ Lanzhou University, Lanzhou 730000, People's Republic of China

${ }^{33}$ Liaoning Normal University, Dalian 116029, People's Republic of China

${ }^{34}$ Liaoning University, Shenyang 110036, People's Republic of China

${ }^{35}$ Nanjing Normal University, Nanjing 210023, People's Republic of China

${ }^{36}$ Nanjing University, Nanjing 210093, People's Republic of China

${ }^{37}$ Nankai University, Tianjin 300071, People's Republic of China

${ }^{38}$ Peking University, Beijing 100871, People's Republic of China

${ }^{39}$ Qufu Normal University, Qufu 273165, People's Republic of China

${ }^{40}$ Shandong Normal University, Jinan 250014, People's Republic of China

${ }^{41}$ Shandong University, Jinan 250100, People's Republic of China

${ }^{42}$ Shanghai Jiao Tong University, Shanghai 200240, People's Republic of China

${ }^{43}$ Shanxi Normal University, Linfen 041004, People's Republic of China

${ }^{44}$ Shanxi University, Taiyuan 030006, People's Republic of China

${ }^{45}$ Sichuan University, Chengdu 610064, People's Republic of China

${ }^{46}$ Soochow University, Suzhou 215006, People's Republic of China

${ }^{47}$ Southeast University, Nanjing 211100, People's Republic of China 


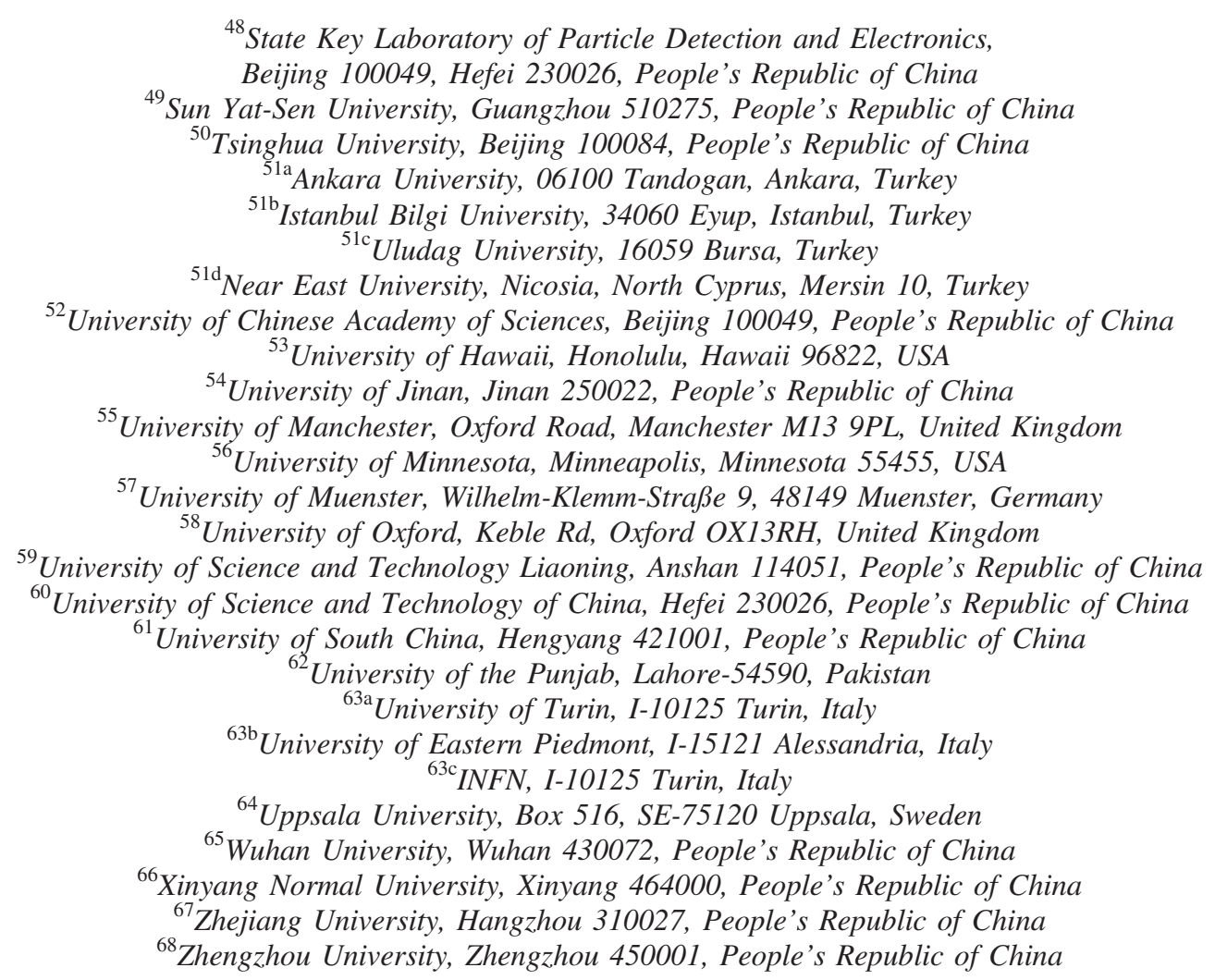

(Received 24 February 2020; accepted 3 April 2020; published 20 April 2020)

\begin{abstract}
We report the first observation of the semimuonic decay $D^{+} \rightarrow \omega \mu^{+} \nu_{\mu}$ using an $e^{+} e^{-}$collision data sample corresponding to an integrated luminosity of $2.93 \mathrm{fb}^{-1}$ collected with the BESIII detector at a center-of-mass energy of $3.773 \mathrm{GeV}$. The absolute branching fraction of the $D^{+} \rightarrow \omega \mu^{+} \nu_{\mu}$ decay is measured to be $\mathcal{B}_{D^{+} \rightarrow \omega \mu^{+} \nu_{\mu}}=\left(17.7 \pm 1.8_{\text {stat }} \pm 1.1_{\text {syst }}\right) \times 10^{-4}$. Its ratio with the world average value of the branching fraction of the $D^{+} \rightarrow \omega e^{+} \nu_{e}$ decay probes lepton flavor universality and it is determined to be $\mathcal{B}_{D^{+} \rightarrow \omega \mu^{+} \nu_{\mu}} / \mathcal{B}_{D^{+} \rightarrow \omega e^{+} \nu_{e}}^{\mathrm{PDG}}=1.05 \pm 0.14$, in agreement with the standard model expectation within one standard deviation.
\end{abstract}

DOI: 10.1103/PhysRevD.101.072005

\footnotetext{
${ }^{a}$ Also at Ankara University, 06100 Tandogan, Ankara, Turkey.

${ }^{\mathrm{b}}$ Also at Bogazici University, 34342 Istanbul, Turkey.

${ }^{c}$ Also at the Moscow Institute of Physics and Technology, Moscow 141700, Russia.

${ }^{\mathrm{d}}$ Also at the Functional Electronics Laboratory, Tomsk State University, Tomsk 634050, Russia.

${ }^{\mathrm{e}}$ Also at the Novosibirsk State University, Novosibirsk 630090, Russia.

${ }^{\mathrm{f}}$ Also at the NRC "Kurchatov Institute", PNPI, 188300 Gatchina, Russia.

${ }^{\mathrm{g}}$ Also at Istanbul Arel University, 34295 Istanbul, Turkey.

${ }^{\mathrm{h}}$ Also at Goethe University Frankfurt, 60323 Frankfurt am Main, Germany.

iAlso at Key Laboratory for Particle Physics, Astrophysics and Cosmology, Ministry of Education; Shanghai Key Laboratory for Particle Physics and Cosmology; Institute of Nuclear and Particle Physics, Shanghai 200240, People's Republic of China.

${ }^{\mathrm{j}}$ Also at Key Laboratory of Nuclear Physics and Ion-beam Application (MOE) and Institute of Modern Physics, Fudan University, Shanghai 200443, People's Republic of China.

${ }^{\mathrm{k}}$ Also at Harvard University, Department of Physics, Cambridge, Massachusetts 02138, USA.

${ }^{1}$ Currently at: Institute of Physics and Technology, Peace Ave.54B, Ulaanbaatar 13330, Mongolia.

${ }^{\mathrm{m}}$ Also at State Key Laboratory of Nuclear Physics and Technology, Peking University, Beijing 100871, People's Republic of China.

${ }^{\mathrm{n}}$ School of Physics and Electronics, Hunan University, Changsha 410082, China.
}

Published by the American Physical Society under the terms of the Creative Commons Attribution 4.0 International license. Further distribution of this work must maintain attribution to the author(s) and the published article's title, journal citation, and DOI. Funded by $S C O A P^{3}$. 
Lepton flavor universality (LFU) is one of the key predictions in the standard model. It requires that the couplings between three generation leptons and gauge bosons are equal with each other. Studies of the semileptonic (SL) decays of pseudoscalar mesons are powerful to test LFU. In recent years, the difference between the measured branching fraction $(\mathrm{BF}) \mathrm{BF}$ ratio $\mathcal{R}_{\tau / \ell}=$ $\mathcal{B}_{B \rightarrow \bar{D}^{(*)} \tau^{+} \nu_{\tau}} / \mathcal{B}_{B \rightarrow \bar{D}^{(*)} \ell^{+} \nu_{\ell}}(\ell=\mu, e)$ [1-7] and the standard model predictions is found to be larger than three standard deviations [8]. Possible physics mechanisms $[9,10]$ were proposed to explain this tension. Comprehensive tests of $e-\mu$ LFU with SL $D$ decays, especially for those lesserknown decays, offer critical information for thorough exploration of LFU.

In 2018, BESIII reported tests of LFU with $D \rightarrow \pi \ell^{+} \nu_{\ell}$ decays [11] which are mediated via $c \rightarrow d \ell^{+} \nu_{\ell}$. The difference between the $\mathrm{BF}$ ratio $\mathcal{R}_{\mu / e}^{c \rightarrow d}=\mathcal{B}_{D \rightarrow \pi \mu^{+} \nu_{\mu}} /$ $\mathcal{B}_{D \rightarrow \pi e^{+} \nu_{e}}$ [12] and the SM prediction is found to be greater than one standard deviation. Tests of LFU with other SL $D$ decays mediated via $c \rightarrow d \ell^{+} \nu_{\ell}$ are important to understand this situation. One possible candidate decay is $D^{+} \rightarrow \omega \mu^{+} \nu_{\mu}$. Although this decay was theoretically predicted before 1990 [13], it has never been experimentally confirmed yet to date. Since 2015, different theoretical approaches, e.g., light-front quark model (LFQM) [14], recalled chiral unitary approach $(\chi \mathrm{UA})[15]$, covariant confined quark model (CCQM) [16], light-cone QCD sum rules (LCSR) [17,18], and relativistic quark model (RQM) [19], were adopted to investigate $D^{+} \rightarrow \omega \mu^{+} \nu_{\mu}$. The predicted BFs range between $(1.78-2.46) \times 10^{-3}$ [14-19], corresponding to the $\mathrm{BF}$ ratios $\mathcal{B}_{D^{+} \rightarrow \omega \mu^{+} \nu_{\mu}}$ / $\mathcal{B}_{D^{+} \rightarrow \omega e^{+} \nu_{e}}$ of (0.93-0.99). Observation and measurement of the BF of $D^{+} \rightarrow \omega \mu^{+} \nu_{\mu}$ are crucial to test $e-\mu \mathrm{LFU}$ with $D^{+} \rightarrow \omega \ell^{+} \nu_{\ell}$ decays. The measured BF are also important to distinguish between various theoretical calculations, thereby improving understanding of nonperturbative effects in heavy meson decays [20,21].

This paper reports the first observation and $\mathrm{BF}$ measurement of $D^{+} \rightarrow \omega \mu^{+} \nu_{\mu}$ as well as a test of $e-\mu \mathrm{LFU}$ with $D^{+} \rightarrow \omega \ell^{+} \nu_{\ell}$ decays, by analyzing $2.93 \mathrm{fb}^{-1}$ of data accumulated with the BESIII detector at a center-of-mass energy $\sqrt{s}=3.773 \mathrm{GeV}$ [22]. Throughout this paper, charge conjugated channels are implied.

The BESIII detector is a magnetic spectrometer [23] located at the Beijing Electron Positron Collider (BEPCII) [24]. The cylindrical core of the BESIII detector consists of a helium-based main drift chamber (MDC), a plastic scintillator time-of-flight system (TOF), and a CsI(Tl) electromagnetic calorimeter (EMC), which are all enclosed in a superconducting solenoidal magnet providing a $1.0 \mathrm{~T}$ magnetic field. The solenoid is supported by an octagonal flux-return yoke with resistive plate counter muon identifier modules interleaved with steel. The acceptance of charged particles and photons is $93 \%$ over $4 \pi$ solid angle.
At $1 \mathrm{GeV} / c$, the charged-particle momentum resolution is $0.5 \%$, and the $d E / d x$ resolution is $6 \%$ for electrons from Bhabha scattering. The EMC measures photon energies with a resolution of $2.5 \%(5 \%)$ at $1 \mathrm{GeV}$ in the barrel (end cap) region. The time resolution of the TOF barrel part is $68 \mathrm{ps}$, while that of the end cap part is $110 \mathrm{ps}$. More details about the BESIII detector are described in Ref. [23].

Simulated samples produced with the GEANT4-based [25] Monte Carlo (MC) software, which includes the geometric description $[26,27]$ of the BESIII detector and the detector response, are used to determine the detection efficiency and to estimate the backgrounds. The simulation includes the beam-energy spread and initial-state radiation (ISR) in the $e^{+} e^{-}$annihilations modeled with the generator KKMC [28]. The inclusive MC samples consist of the production of the $D \bar{D}$ pairs, the non- $D \bar{D}$ decays of the $\psi(3770)$, the ISR production of the $J / \psi$ and $\psi(3686)$ states, and the continuum processes $\left(e^{+} e^{-} \rightarrow q \bar{q},(q=u, d, s)\right)$ incorporated in KKMC [28]. The known decay modes are modeled with EVTGEN [29] using BFs taken from the Particle Data Group (PDG) [12], and the remaining unknown decays from the charmonium states with LUNDCHARM [30]. The final-state radiation from charged final-state particles is incorporated with the PHOTOS package [31]. The $D^{+} \rightarrow$ $\omega \mu^{+} \nu_{\mu}$ decay is simulated by a model with the form factor parameters of $r_{V}=V(0) / A_{1}(0)=1.24 \pm 0.11$ and $r_{2}=A_{2}(0) / A_{1}(0)=1.06 \pm 0.16$, which are quoted from Ref. [32].

At $\sqrt{s}=3.773 \mathrm{GeV}$, the $\psi(3770)$ resonance decays predominately into $D^{0} \bar{D}^{0}$ or $D^{+} D^{-}$meson pairs. The $D^{-}$mesons are reconstructed by their hadronic decays to $K^{+} \pi^{-} \pi^{-}, K_{S}^{0} \pi^{-}, K^{+} \pi^{-} \pi^{-} \pi^{0}, K_{S}^{0} \pi^{-} \pi^{0}, K_{S}^{0} \pi^{+} \pi^{-} \pi^{-}$, and $K^{+} K^{-} \pi^{-}$, and referred to as single-tag (ST) $D^{-}$mesons. In the sides recoiling against of the ST $D^{-}$mesons, the candidate $D^{+} \rightarrow \omega \mu^{+} \nu_{\mu}$ decays are selected to form double-tag (DT) events. The absolute BF of $D^{+} \rightarrow \omega \mu^{+} \nu_{\mu}$ is determined by

$$
\mathcal{B}_{\mathrm{SL}}=N_{\mathrm{DT}} /\left(N_{\mathrm{ST}}^{\mathrm{tot}} \cdot \varepsilon_{\mathrm{SL}} \cdot \mathcal{B}_{\omega} \cdot \mathcal{B}_{\pi^{0}}\right),
$$

where $N_{\mathrm{ST}}^{\text {tot }}$ and $N_{\mathrm{DT}}$ are the ST and DT yields in the data sample, $\mathcal{B}_{\omega}$ and $\mathcal{B}_{\pi^{0}}$ are the BFs of the $\omega \rightarrow \pi^{+} \pi^{-} \pi^{0}$ and $\pi^{0} \rightarrow \gamma \gamma$ decays, respectively, and $\varepsilon_{\mathrm{SL}}=\Sigma_{i}\left[\left(\varepsilon_{\mathrm{DT}}^{i}\right.\right.$. $\left.\left.N_{\mathrm{ST}}^{i}\right) /\left(\varepsilon_{\mathrm{ST}}^{i} \cdot N_{\mathrm{ST}}^{\mathrm{tot}}\right)\right]$ is the efficiency of detecting the SL decay in the presence of the ST $D^{-}$meson. Here $i$ denotes the tag mode, and $\varepsilon_{\mathrm{ST}}$ and $\varepsilon_{\mathrm{DT}}$ are the efficiencies of selecting the ST and DT candidates, respectively.

The same selection criteria as reported in Refs. [11, 33-36] are used in this analysis. Charged tracks are required to have polar angle $(\theta)$ within $|\cos \theta|<0.93$, and except for those from $K_{S}^{0}$ decays, are required to originate from an interaction region defined by $\left|V_{x y}\right|<$ $1 \mathrm{~cm}$ and $\left|V_{z}\right|<10 \mathrm{~cm}$, where $\left|V_{x y}\right|$ and $\left|V_{z}\right|$ refer to the distances of closest approach of the reconstructed track to 
the interaction point in the $x y$ plane and the $z$ direction (along the beam), respectively.

Particle identification (PID) of charged kaons and pions is implemented with the $d E / d x$ and TOF information. For muon identification, the EMC information is also included. For each charged track, the combined confidence levels for the electron, muon, pion, and kaon hypotheses $\left(C L_{e}\right.$, $C L_{\mu}, C L_{\pi}$, and $C L_{K}$ ) are calculated. The charged tracks satisfying $C L_{K(\pi)}>C L_{\pi(K)}$ are identified as kaon (pion) candidates. The muon candidates are required to satisfy $C L_{\mu}>0.001, C L_{\mu}>C L_{e}$, and $C L_{\mu}>C L_{K}$, and their deposited energy in the EMC is required to be within $(0.15,0.25) \mathrm{GeV}$ to suppress backgrounds misidentified from charged hadrons.

The $K_{S}^{0}$ candidates are selected from pairs of opposite charged tracks with $\left|V_{z}\right|<20 \mathrm{~cm}$, but without requirements on $\left|V_{x y}\right|$. The two tracks are designated as pions without PID requirements, constrained to a common vertex and required to have an invariant mass satisfying $\left|M_{\pi^{+} \pi^{-}}-m_{K_{S}^{0}}\right|<12 \mathrm{MeV} / c^{2}$, where $m_{K_{S}^{0}}$ is the $K_{S}^{0}$ nominal mass [12]. The selected $K_{S}^{0}$ candidate must have a decay length greater than two times the vertex resolution.

Photon candidates are selected using EMC information. It is required that the shower time is within $700 \mathrm{~ns}$ of the event start time, the shower energy must be greater than 25 (50) $\mathrm{MeV}$ in the barrel (end cap) region [23], and the opening angle between the candidate shower and any charged tracks must be greater than $10^{\circ}$.

The $\pi^{0}$ candidates are selected from photon pairs with invariant mass within $(0.115,0.150) \mathrm{GeV} / c^{2}$. To improve the momentum resolution, a one constraint (1-C) kinematic fit is performed constraining the pair's $\gamma \gamma$ invariant mass to the $\pi^{0}$ nominal mass [12], and the $\chi_{1-\mathrm{C}}^{2}$ of the 1-C (massconstraint) kinematic fit is required to be less than 200 .

The energy difference $(\Delta E)$ and beam-constrained mass $\left(M_{\mathrm{BC}}\right)$ are used to select ST $D^{-}$candidates, where

$$
\Delta E \equiv E_{D^{-}}-E_{\text {beam }}
$$

and

$$
M_{\mathrm{BC}} \equiv \sqrt{E_{\text {beam }}^{2}-\left|\vec{p}_{D^{-}}\right|^{2}}
$$

$E_{\text {beam }}$ is the beam energy, and $\vec{p}_{D^{-}}$and $E_{D^{-}}$are the total momentum and energy of the ST candidate calculated in the $e^{+} e^{-}$rest frame, respectively. The $D^{-}$candidates are expected to concentrate around zero in the $\Delta E$ distribution and around the nominal $D^{-}$mass in the $M_{\mathrm{BC}}$ distribution. For each tag mode, the one with minimum $|\Delta E|$ is retained. Combinatorial backgrounds in the $M_{\mathrm{BC}}$ distributions are suppressed with a requirement of $\Delta E \in(-0.055,0.045) \mathrm{GeV}$ for tags containing $\pi^{0}$ and $\Delta E \in(-0.025,0.025) \mathrm{GeV}$ for other tags.
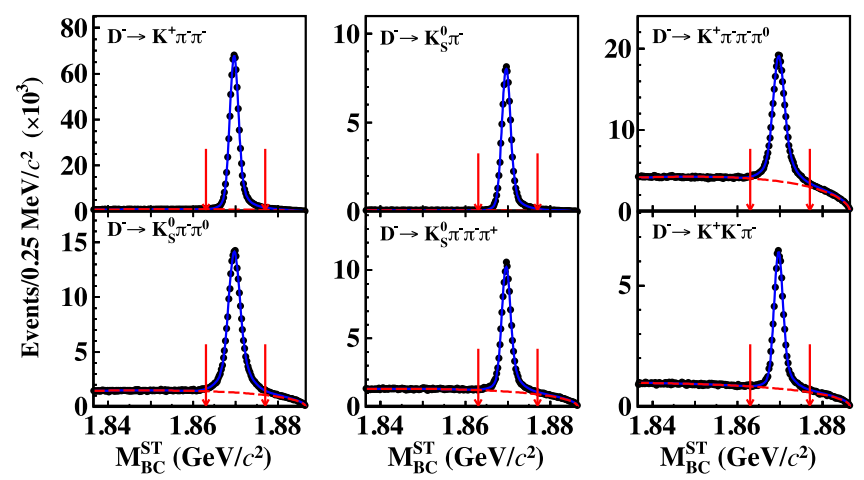

FIG. 1. Fits to the $M_{\mathrm{BC}}$ distributions of the ST candidate events. The dots with error bars are data, the blue solid curves are the fit results, the red dashed curves are the fitted backgrounds, and the pair of red arrows in each subfigure denote the ST $D^{-}$signal region.

For each tag mode, the ST yield is determined by fitting the $M_{\mathrm{BC}}$ distribution of the candidates surviving all above requirements. In the fit, the $D^{-}$signal is modeled with a shape obtained from an MC simulation convolved with a double Gaussian describing the difference between data and MC simulations, and the combinatorial background is described by an ARGUS function [37]. The resulting fits to the $M_{\mathrm{BC}}$ distributions for each mode are shown in Fig. 1. Candidates in the $M_{\mathrm{BC}}$ signal region, $(1.863,1.877) \mathrm{GeV} / c^{2}$, are kept for further analysis. The ST yields in data and the ST efficiencies for individual tags are shown in Table I. Summing over the ST yields for all tags gives a total yield of $N_{\mathrm{ST}}^{\text {tot }}=1522474 \pm 2215$, where the uncertainty is statistical.

The $D^{+} \rightarrow \omega \mu^{+} \nu_{\mu}$ candidates are selected from the remaining charged tracks and photons that have not been used for the ST reconstruction. Each candidate must have three good charged tracks and one $\pi^{0}$ candidate. If there are multiple neutral pions, the one with the minimum $\chi_{1-\mathrm{C}}^{2}$ is chosen. One of the three charged tracks must be identified as a muon, and the other two as $\pi^{+} \pi^{-}$. The total charge of the DT event is required to be zero. The $\omega$ candidates are selected from $\pi^{+} \pi^{-} \pi^{0}$ combinations, and we require

TABLE I. Summary of ST yields $\left(N_{\mathrm{ST}}^{i}\right)$, ST efficiencies $\left(\varepsilon_{\mathrm{ST}}^{i}\right)$ and DT efficiencies $\left(\varepsilon_{\mathrm{DT}}^{i}\right)$ for different tag modes. Uncertainties are statistical only. Efficiencies do not include the BFs of $K_{S}^{0} \rightarrow \pi^{+} \pi^{-}, \pi^{0} \rightarrow \gamma \gamma$, and $\omega \rightarrow \pi^{+} \pi^{-} \pi^{0}$.

\begin{tabular}{lccc}
\hline \hline Tag mode & $N_{\mathrm{ST}}^{i}$ & $\epsilon_{\mathrm{ST}}^{i}(\%)$ & $\epsilon_{\mathrm{DT}}^{i}(\%)$ \\
\hline$D^{-} \rightarrow K^{+} \pi^{-} \pi^{-}$ & $782669 \pm 990$ & $50.61 \pm 0.06$ & $4.28 \pm 0.05$ \\
$D^{-} \rightarrow K_{S}^{0} \pi^{-}$ & $91345 \pm 320$ & $50.41 \pm 0.17$ & $4.57 \pm 0.06$ \\
$D^{-} \rightarrow K^{+} \pi^{-} \pi^{-} \pi^{0}$ & $251008 \pm 1135$ & $26.74 \pm 0.09$ & $1.89 \pm 0.04$ \\
$D^{-} \rightarrow K_{S}^{0} \pi^{-} \pi^{0}$ & $215364 \pm 1238$ & $27.29 \pm 0.07$ & $2.26 \pm 0.06$ \\
$D^{-} \rightarrow K_{S}^{0} \pi^{+} \pi^{-} \pi^{-}$ & $113054 \pm 889$ & $28.29 \pm 0.12$ & $2.16 \pm 0.09$ \\
$D^{-} \rightarrow K^{+} K^{-} \pi^{-}$ & $69034 \pm 460$ & $40.87 \pm 0.24$ & $3.05 \pm 0.05$ \\
\hline \hline
\end{tabular}




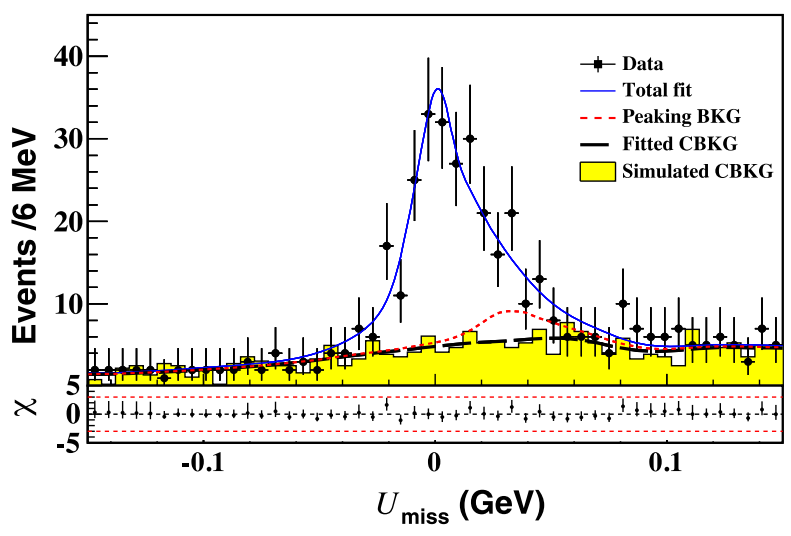

FIG. 2. The results of a fit to the $U_{\text {miss }}$ distribution of the $D^{+} \rightarrow \omega \mu^{+} \nu_{\mu}$ candidate events. The dots with error bars are data and the blue solid curve is the fit result. The yellow hatched histogram is the MC-simulated combinatorial background ( $\mathrm{Si}$ mulated CBKG), the black dashed curve is the result of a fit of the combinatorial background (Fitted $\mathrm{CBKG}$ ), and the difference between the red dotted and black dashed curves is the peaking background of $D^{+} \rightarrow \omega \pi^{+} \pi^{0}$ (Peaking BKG). The bottom plot shows the $\chi$ distribution obtained from the fit.

$\left|M_{\pi^{+} \pi^{-} \pi^{0}}-m_{\omega}\right|<0.025 \mathrm{GeV} / c^{2}$, where $m_{\omega}$ is the $\omega$ nominal mass [12] and $M_{\pi^{+} \pi^{-} \pi^{0}}$ is the invariant mass of the $\pi^{+} \pi^{-} \pi^{0}$ combination. If two $\pi^{+} \pi^{-} \pi^{0}$ combinations can be formed due to mis-identification between $\pi^{+}$and $\mu^{+}$, the one with $M_{\pi^{+} \pi^{-} \pi^{0}}$ closer to $m_{\omega}$ is kept as the $\omega$ candidate. To suppress backgrounds from the SL decays $D^{+} \rightarrow$ $\bar{K}^{*}(892)^{0} \mu^{+} \nu_{\mu}$ with $\bar{K}^{*}(892)^{0} \rightarrow K_{S}^{0}\left(\pi^{+} \pi^{-}\right) \pi^{0}$, we require $\left|M_{\pi^{+} \pi^{-}}-m_{K_{S}^{0}}\right|>0.015 \mathrm{GeV} / c^{2}$ and $\left|M_{\pi_{\mu \rightarrow \pi}^{+} \pi^{-}}-m_{K_{S}^{0}}\right|>$ $0.015 \mathrm{GeV} / c^{2}$, where $M_{\pi^{+} \pi^{-}}$and $M_{\pi_{\mu \rightarrow \pi}^{+} \pi^{-}}$are the invariant masses of the $\pi^{+} \pi^{-}$and $\mu^{+} \pi^{-}$combinations, respectively; and $\pi_{\mu \rightarrow \pi}^{+}$denotes that the mass of the muon candidate has been replaced by the $\pi^{+}$mass. These requirements correspond to approximately four times the fitted mass resolution of $K_{S}^{0}$ around its nominal mass. To suppress backgrounds from the hadronic decays $D^{+} \rightarrow K_{S}^{0}\left(\pi^{0} \pi^{0}\right) \pi^{+} \pi^{+} \pi^{-}$, the invariant mass of the system recoiling against the $D^{-} \pi_{\mu \rightarrow \pi}^{+} \pi^{+} \pi^{-}$combination $\left(M_{D^{-} \pi_{\mu \rightarrow \pi}^{+} \pi^{+} \pi^{-}}^{\text {recil }}\right)$ is required to be outside the range of $(0.45,0.55) \mathrm{GeV} / c^{2}$. The peaking backgrounds from the hadronic decays $D^{+} \rightarrow \omega \pi^{+}$and $D^{+} \rightarrow \omega \pi^{+} \pi^{0}$ are suppressed by requiring $M_{\omega \mu^{+}}<$ $1.5 \mathrm{GeV} / c^{2}$ and $E_{\text {extra }}^{\max }<0.15 \mathrm{GeV}$. Here, $M_{\omega \mu^{+}}$is the invariant mass of the $\omega \mu^{+}$combination and $E_{\mathrm{extra}}^{\max }$ is the maximum energy of any photon that is not used in the DT selection.

The neutrino of the SL $D$ decay is undetectable by the BESIII detector. The information of the $D^{+} \rightarrow \omega \mu^{+} \nu_{\mu}$ decay is inferred by the difference between the missing energy $\left(E_{\text {miss }}\right)$ and the missing momentum $\left(\left|\vec{p}_{\text {miss }}\right|\right)$ of the observed particles of the DT event calculated in the $e^{+} e^{-}$center-of-mass frame, $U_{\text {miss }} \equiv E_{\text {miss }}-\left|\vec{p}_{\text {miss }}\right|$. Here, $E_{\text {miss }} \equiv E_{\text {beam }}-E_{\omega}-E_{\mu^{+}}$and $\vec{p}_{\text {miss }} \equiv \vec{p}_{D^{+}}-\vec{p}_{\omega}-\vec{p}_{\mu^{+}}$, where $E_{\omega\left(\mu^{+}\right)}$and $\vec{p}_{\omega\left(\mu^{+}\right)}$are the energy and momentum of the $\omega\left(\mu^{+}\right)$candidates, respectively. The $U_{\text {miss }}$ resolution is improved by constraining the $D^{+}$energy and momentum with the beam energy and $\vec{p}_{D^{+}}=-\hat{p}_{D^{-}} \sqrt{E_{\text {beam }}^{2}-m_{D^{-}}^{2}}$, where $\hat{p}_{D^{-}}$is the unit vector in the momentum direction of the tagged $D^{-}$and $m_{D^{-}}$is the $D^{-}$nominal mass [12].

The $U_{\text {miss }}$ distribution of the accepted DT events of data is shown in Fig. 2. An unbinned maximum likelihood fit to this distribution is used to determine the SL decay yield. The shapes of all the components in the fit are obtained from MC simulations, including the SL signal, the peaking background from the hadronic decays $D^{+} \rightarrow \omega \pi^{+} \pi^{0}$, and other backgrounds, while their yields are left free. The number of $D^{+} \rightarrow \omega \mu^{+} \nu_{\mu}$ decays obtained is $N_{\mathrm{DT}}=194 \pm 20$, where the uncertainty is statistical.

The fourth column of Table I lists the DT efficiencies for individual tag modes. The signal efficiency weighted by the ST yields in data is $\varepsilon_{\mathrm{SL}}=(8.15 \pm 0.07) \%$. Detailed studies show that the momentum and $\cos \theta$ distributions of $\omega$ and $\mu^{+}$of data are modeled well by MC simulations. The $\mathrm{BF}$ of the $D^{+} \rightarrow \omega \mu^{+} \nu_{\mu}$ decay is obtained by Eq. (1) to be

$$
\mathcal{B}_{D^{+} \rightarrow \omega \mu^{+} \nu_{\mu}}=(17.7 \pm 1.8 \pm 1.1) \times 10^{-4},
$$

where the first uncertainty is statistical and the second systematic.

With the DT method, most systematic uncertainties arising from the ST side cancel. In the BF measurement, the systematic uncertainties arise from the following sources. The uncertainty in the total ST yield, which is mainly from the uncertainty due to the $M_{\mathrm{BC}}$ fit of the ST candidates, has been studied in Refs. [11,33,34] and is assigned as $0.5 \%$. The tracking and PID efficiencies of the pion and muon are studied by analyzing the DT hadronic $D \bar{D}$ events and $e^{+} e^{-} \rightarrow \gamma \mu^{+} \mu^{-}$events, respectively. The systematic uncertainties associated with the pion tracking (PID), muon tracking (PID) are assigned to be $0.2 \%(0.3 \%)$

TABLE II. Comparison of the BFs between $D^{+} \rightarrow \omega e^{+} \nu_{e}$ and $D^{+} \rightarrow \omega \mu^{+} \nu_{\mu}$.

\begin{tabular}{|c|c|c|c|c|c|c|c|}
\hline & CCQM [14] & $\chi \mathrm{UA}[15]$ & LFQM [16] & LCSR [17] & LCSR [18] & RQM [19] & Measurement \\
\hline $\mathcal{B}_{D^{+} \rightarrow \omega \mu^{+} \nu_{\mu}}\left(\times 10^{-4}\right)$ & 17.8 & 22.9 & $20 \pm 2$ & $18.5_{-1.3}^{+1.9}$ & $17.3_{-4.0}^{+4.8}$ & 20.8 & $17.7 \pm 1.8 \pm 1.1$ \\
\hline $\mathcal{B}_{D^{+} \rightarrow \omega e^{+} \nu_{e}}\left(\times 10^{-4}\right)$ & 18.5 & 24.6 & $21 \pm 2$ & $19.3_{-1.4}^{+2.0}$ & $17.4_{-4.0}^{+4.8}$ & 21.7 & $16.9 \pm 1.1[12]$ \\
\hline $\mathcal{B}_{D^{+} \rightarrow \omega \mu^{+} \nu_{\mu}} / \mathcal{B}_{D^{+} \rightarrow \omega e^{+} \nu_{\mu}}^{\mathrm{PDG}}$ & 0.96 & 0.93 & 0.95 & 0.96 & 0.99 & 0.96 & $1.05 \pm 0.14$ \\
\hline
\end{tabular}


and $0.3 \%(0.3 \%)$, respectively. The $\pi^{0}$ efficiency, including effects of photon selection, the 1-C kinematic fit, and the mass window, is studied with DT hadronic $D \bar{D}$ decays [33,34], and a systematic uncertainty of $0.7 \%$ is assigned to each $\pi^{0}$. The uncertainty of the $E_{\text {extray }}^{\max }$ requirement is estimated to be $4.4 \%$ by analyzing the DT $D \bar{D}$ events of $D^{+} \rightarrow \omega\left(\pi^{+} \pi^{-} \pi^{0}\right) e^{+} \nu_{e}, \quad D^{+} \rightarrow$ $K_{S}^{0}\left(\pi^{+} \pi^{-}\right) \pi^{0} e^{+} \nu_{e}, \quad D^{+} \rightarrow K_{S}^{0}\left(\pi^{+} \pi^{-}\right) e^{+} \nu_{e}, \quad$ and $\quad D^{+} \rightarrow$ $K_{S}^{0}\left(\pi^{+} \pi^{-}\right) \pi^{+} \pi^{0}$. The uncertainties due to the $M_{\omega \mu^{+}}$ requirement and the $K_{S}^{0}$ rejection $\left(M_{\pi^{+} \pi^{-}}, M_{\pi_{\mu \rightarrow \pi}^{+} \pi^{-}}\right.$, and $M_{D^{-} \pi_{\mu \rightarrow \pi}^{+} \pi^{+} \pi^{-}}^{\text {recoli }}$ ) are evaluated by repeating measurements varying the nominal requirements by $\pm 0.05 \mathrm{GeV} / c^{2}$ and $\pm 0.005 \mathrm{GeV} / c^{2}$, respectively, and they are found to be negligible. The uncertainty originating from the $U_{\text {miss }}$ fit is assigned to be $3.4 \%$, which is estimated with alternative fit ranges and signal and background shapes. The uncertainty due to the limited MC size is $0.5 \%$. The uncertainty in the MC model is assigned to be $2.3 \%$, by comparing our nominal DT efficiency with one obtained using an ISGW model [20]. All these systematic uncertainties are assumed to be independent, and their quadratic sum gives a total systematic uncertainty of $6.3 \%$.

To summarize, by analyzing the data sample with an integrated luminosity of $2.93 \mathrm{fb}^{-1}$ collected at $\sqrt{s}=$ $3.773 \mathrm{GeV}$ with the BESIII detector, we report the first observation and $\mathrm{BF}$ measurement of the SL decay $D^{+} \rightarrow \omega \mu^{+} \nu_{\mu}$. Table II shows the comparison of our BF to various theoretical calculations of $D^{+} \rightarrow \omega \mu^{+} \nu_{\mu}$ decay. Our BF is consistent with the predicted values based on the LFQM, CCQM, and LCSR methods [14,16-18], but differs from those based on the $\chi \mathrm{UA}$ [15] and RQM [19] methods by $2.5 \sigma$ and $1.5 \sigma$, respectively. Combining the $\mathcal{B}_{D^{+} \rightarrow \omega \mu^{+} \nu_{\mu}}$ measured in this work with the world average $\mathcal{B}_{D^{+} \rightarrow \omega e^{+} \nu_{e}}^{\text {PDG }}=(16.9 \pm 1.1) \times 10^{-4} \quad[12,32,38]$, we obtain the $\mathrm{BF}$ ratio to be $\mathcal{B}_{D^{+} \rightarrow \omega \mu^{+} \nu_{\mu}} / \mathcal{B}_{D^{+} \rightarrow \omega e^{+} \nu_{e}}^{\mathrm{PDG}}=1.05 \pm 0.14$.
It agrees with the standard model predictions (0.93-0.99) $[14-17,19]$ within uncertainties, and implies no violation of $e-\mu \mathrm{LFU}$ found with current statistics.

\section{ACKNOWLEDGMENTS}

The BESIII collaboration thanks the staff of BEPCII and the IHEP computing center for their strong support. This work is supported in part by National Key Basic Research Program of China under Contract No. 2015CB856700; National Natural Science Foundation of China (NSFC) under Contracts No. 11675200, No. 11625523, No. 11635010, No. 11735014, No. 11822506, No. 11835012, No. 11961141012; the Chinese Academy of Sciences (CAS) Large-Scale Scientific Facility Program; Joint Large-Scale Scientific Facility Funds of the NSFC and CAS under Contracts No. U1632109, No. U1532257, No. U1532258, No. U1732263, No. U1832207; CAS Key Research Program of Frontier Sciences under Contracts No. QYZDJ-SSW-SLH003, No. QYZDJ-SSW-SLH040; 100 Talents Program of CAS; Institute of Nuclear and Particle Physics, Astronomy and Cosmology (INPAC) and Shanghai Key Laboratory for Particle Physics and Cosmology; ERC under Contract No. 758462; German Research Foundation DFG under Contracts No. Collaborative Research Center CRC 1044 and No. FOR 2359; Istituto Nazionale di Fisica Nucleare, Italy; Ministry of Development of Turkey under Contract No. DPT2006K-120470; National Science and Technology fund; STFC (United Kingdom); The Knut and Alice Wallenberg Foundation (Sweden) under Contract No. 2016.0157; The Royal Society, UK under Contracts No. DH140054 and No. DH160214; The Swedish Research Council; U.S. Department of Energy under Contracts No. DE-FG02-05ER41374, No. DE-SC0010118, No. DE-SC-0012069.
[1] J. P. Lees et al. (BABAR Collaboration), Phys. Rev. Lett. 109, 101802 (2012).

[2] J. P. Lees et al. (BABAR Collaboration), Phys. Rev. D 88, 072012 (2013).

[3] R. Aaij et al. (LHCb Collaboration), Phys. Rev. Lett. 115, 111803 (2015).

[4] M. Huschle et al. (Belle Collaboration), Phys. Rev. D 92, 072014 (2015).

[5] Y. Sato et al. (Belle Collaboration), Phys. Rev. D 94, 072007 (2016).

[6] R. Aaij et al. (LHCb Collaboration), Phys. Rev. D 97, 072013 (2018).
[7] A. Abdesselam et al. (Belle Collaboration), arXiv:1904 .08794 .

[8] Y. Amhis et al. (Heavy Flavor Averaging Group), arXiv: 1909.12524.

[9] S. Fajfer, I. Nisandzic, and U. Rojec, Phys. Rev. D 91, 094009 (2015).

[10] G. C. Branco, P. M. Ferreira, L. Lavoura, M. N. Rebelo, M. Sher, and J. P. Silva, Phys. Rep. 516, 1 (2012).

[11] M. Ablikim et al. (BESIII Collaboration), Phys. Rev. Lett. 121, 171803 (2018).

[12] M. Tanabashi et al. (Particle Data Group), Phys. Rev. D 98, 030001 (2018), and 2019 update. 
[13] N. Isgur, D. Scora, B. Grinstein, and M. B. Wise, Phys. Rev. D 39, 799 (1989).

[14] H. Y. Cheng and X. W. Kang, Eur. Phys. J. C 77, 587 (2017); 77, 863(E) (2017).

[15] T. Sekihara and E. Oset, Phys. Rev. D 92, 054038 (2015).

[16] N. R. Soni, M. A. Ivanov, J. G. Körner, J. N. Pandya, P. Santorelli, and C. T. Tran, Phys. Rev. D 98, 114031 (2018).

[17] M. A. Ivanov, J. G. Körner, J. N. Pandya, P. Santorelli, N. R. Soni, and C. T. Tran, Front. Phys. 14, 64401 (2019).

[18] H. B. Fu, W. Cheng, L. Zeng, and D. D. Hu, arXiv:2003 .07626.

[19] R. N. Faustov, V. O. Galkin, and X. W. Kang, Phys. Rev. D 101, 013004 (2020).

[20] D. Scora and N. Isgur, Phys. Rev. D 52, 2783 (1995).

[21] M. B. Voloshin, Phys. Lett. B 515, 74 (2001).

[22] M. Ablikim et al. (BESIII Collaboration), Chin. Phys. C 37, 123001 (2013); Phys. Lett. B 753, 629 (2016).

[23] M. Ablikim et al. (BESIII Collaboration), Nucl. Instrum. Methods Phys. Res., Sect. A 614, 345 (2010).

[24] C. H. Yu et al., Proceedings of IPAC2016, Busan, Korea (2016).

[25] S. Agostinelli et al. (GEANT4 Collaboration), Nucl. Instrum. Methods Phys. Res., Sect. A 506, 250 (2003).

[26] Y. Liang, B. Zhu, and Z. Y. You et al., Nucl. Instrum. Methods Phys. Res., Sect. A 603, 325 (2009).
[27] Z. Y. You, Y. T. Liang, and Y. J. Mao, Chin. Phys. C 32, 572 (2008).

[28] S. Jadach, B. F. L. Ward, and Z. Was, Phys. Rev. D 63, 113009 (2001); Comput. Phys. Commun. 130, 260 (2000).

[29] D. J. Lange, Nucl. Instrum. Methods Phys. Res., Sect. A 462, 152 (2001); R. G. Ping, Chin. Phys. C 32, 599 (2008).

[30] J. C. Chen, G. S. Huang, X. R. Qi, D. H. Zhang, and Y. S. Zhu, Phys. Rev. D 62, 034003 (2000); R. L. Yang, R. G. Ping, and H. Chen, Chin. Phys. Lett. 31, 061301 (2014).

[31] E. Richter-Was, Phys. Lett. B 303, 163 (1993).

[32] M. Ablikim et al. (BESIII Collaboration), Phys. Rev. D 92, 071101 (2015).

[33] M. Ablikim et al. (BESIII Collaboration), Eur. Phys. J. C 76, 369 (2016).

[34] M. Ablikim et al. (BESIII Collaboration), Chin. Phys. C 40, 113001 (2016).

[35] M. Ablikim et al. (BESIII Collaboration), Phys. Rev. Lett. 123, 231801 (2019).

[36] M. Ablikim et al. (BESIII Collaboration), Phys. Rev. D 101, 052009 (2020).

[37] H. Albrecht et al. (ARGUS Collaboration), Phys. Lett. B 241, 278 (1990).

[38] S. Dobbs et al. (CLEO Collaboration), Phys. Rev. Lett. 110, 131802 (2013). 